Mathematical Research Letters 2, 783-790 (1995)

\title{
ON THE COHOMOLOGY OF PARABOLIC LINE BUNDLES
}

\author{
INDRANIL BISWAS
}

\section{Introduction}

Let $X$ be a smooth projective variety over $\mathbb{C}$ of dimension $n$. Let $D=\sum_{i=1}^{d} D_{i}$ be a divisor of normal crossing with decomposition into irreducible components. Fix rational numbers $\left\{\alpha_{1}, \ldots, \alpha_{d}\right\}$ with $0<\alpha_{i}<1$. Assume that the Poincare dual of the $\mathbb{Q}$-divisor $\sum_{i=1}^{d} \alpha_{i} D_{i}$ is in the image of $H^{2}(X, \mathbb{Z})$ in $H^{2}(X, \mathbb{Q})$. Such a data constitutes a parabolic bundle in the sense of $[\mathrm{MY}]$. Let $P(X)$ be a component of the moduli space of parabolic bundles of parabolic degree zero (which simply is a component of the Picard group of $X$ consisting of line bundles with first Chern class $-\sum_{i=1}^{d} \alpha_{i}\left[D_{i}\right]$, where $\left[D_{i}\right]$ is the Poincaré dual of $\left.D_{i}\right)$.

Let $\operatorname{Pic}^{0}(X)$ be the abelian variety consisting of isomorphism classes of topologically trivial line bundles. The group $\operatorname{Pic}^{0}(X)$ acts on $P(X)$ using tensor product, and $P(X)$ is an affine group for $\operatorname{Pic}^{0}(X)$.

Define the subvariety

$$
T_{m}^{i}:=\left\{L \in P(X) \mid \operatorname{dim} H^{i}(X, L) \geq m\right\} \subset P(X) .
$$

We prove the following theorem.

Theorem A. Any irreducible component of $T_{m}^{i}$ is a translation of an abelian subvariety of $\mathrm{Pic}^{0}(X)$ by a point of $P(X)$ for the above action.

The special case of the above theorem where $D$ is empty was proved in [GL2].

Let $Y$ be smooth variety on which a finite group $G$ acts, such that the quotient, $Y / G$, is a smooth variety. We first observe that if we consider $G$ invariant part of the cohomology, the result in [GL2] easily extends to the case of the moduli space of the group of topologically trivial line bundles on $Y$ equipped with a lift of the action of $G$.

We now describe the main theme of this work. Using the "covering lemma" of Y. Kawamata, the moduli space $P(X)$ can be identified with the moduli space $G$-equivariant line bundles of the above type for some suitable $Y$ and $G$.

Received September 8, 1995 
Using the above identification we deduce Theorem A from the corresponding result on $G$-equivariant bundles.

\section{Equivariant line bundles and parabolic line bundles}

2a. Group action on a line bundle. Let $Y$ be a connected smooth projective variety over $\mathbb{C}$ of dimension $n$. The group of automorphisms of $Y$ is denoted by $\operatorname{Aut}(Y)$. Let $G$ be a finite group acting faithfully on $Y$. In other words,

$$
\rho: G \longrightarrow \operatorname{Aut}(Y)
$$

is a monomorphism of a finite group $G$ into $\operatorname{Aut}(Y)$.

Definition 2.1. An orbifold line bundle on $Y$ is a line bundle $L$ on $Y$ together with a lift of action of $G$, which means that $G$ acts on the total space of $L$, and for any $g \in G$, the action of $g$ on $L$ is an isomorphism between $L$ and $\rho\left(g^{-1}\right)^{*} L$.

Remark. A line bundle $L$ with the property that for any $g \in G$, the bundle $L$ is isomorphic to $\rho\left(g^{-1}\right)^{*} L$, need not have an orbifold structure. For an orbifold bundle $L^{\prime}$, clearly there is a natural lift of action of $G$ on $H^{0}\left(Y, L^{\prime}\right)$. But an abelian variety together with a power of a principal polarization constitutes an example where a finite group of symmetry (the Heisenberg group) of the line bundle does not lift to the space of sections [M].

Let $\operatorname{Pic}^{0}(Y)$ be the abelian variety parametrizing holomorphic isomorphism classes of topologically trivial line bundles on $Y$. The group $G$ acts on $\operatorname{Pic}^{0}(Y)$ by $g \circ L=\rho\left(g^{-1}\right)^{*} L$. Let $\operatorname{Pic}_{G}(Y) \subset \operatorname{Pic}^{0}(Y)$ be the set of fixed points of this action of $G$. Note that $\operatorname{Pic}_{G}(Y)$ is a complex submanifold of $\operatorname{Pic}^{0}(Y)$; moreover, it is a closed subgroup of the abelian variety $\operatorname{Pic}^{0}(Y)$.

Take any $L \in \operatorname{Pic}_{G}(Y)$. The above remark indicates that $L$ need not have an orbifold structure. We want to identify the obstruction to having an orbifold structure. For $g \in G$ fix an isomorphism

$$
\psi_{g}: L \longrightarrow \rho\left(g^{-1}\right)^{*} L \text {. }
$$

So for $g, h \in G, \psi_{g} \circ \psi_{h} \circ \psi_{(g h)^{-1}}$ is an automorphism of $L$, and hence is a nonzero scalar. The map $G \times G \rightarrow \mathbb{C}^{*}$ defined by

$$
(g, h) \longmapsto \psi_{g} \circ \psi_{h} \circ \psi_{(g h)^{-1}}
$$

gives a 2-cocycle, which we denote by $\psi_{L}$. It is easy to check that the cohomology class $\bar{\psi}_{L}$ represented by $\psi_{L}$, does not depend upon the choices of $\psi_{g}, g \in G$. The line bundle $L$ has an orbifold structure if and only if $\bar{\psi}_{L}=0$.

Let $\operatorname{Pic}_{G}(Y)^{\prime} \subset \operatorname{Pic}^{0}(Y)$ be the subset consisting of all those line bundles which admit an orbifold structure. So we have $\operatorname{Pic}_{G}(Y)^{\prime} \subset \operatorname{Pic}_{G}(Y)$. 
For two orbifold bundles $(L, \bar{\rho})$ and $\left(L^{\prime}, \bar{\rho}^{\prime}\right)$, there is an obvious orbifold

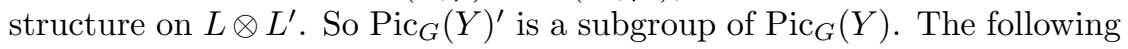
sequence of abelian groups is exact

$$
0 \longrightarrow \operatorname{Pic}_{G}(Y)^{\prime} \longrightarrow \operatorname{Pic}_{G}(Y) \longrightarrow H^{2}\left(G, \mathbb{C}^{*}\right)
$$

where the last homomorphism is given by $L \longmapsto \bar{\psi}_{L}$ described earlier. So $\operatorname{Pic}_{G}(Y)^{\prime}$ is a both open and closed subset of $\operatorname{Pic}_{G}(Y)$, i.e., a union of some components of $\operatorname{Pic}_{G}(Y)$.

Now we want to determine how many distinct orbifold structures a given line bundle admits. Let $(L, \bar{\rho})$ be an orbifold line bundle and $\lambda$ a character of $G$. Then we can construct a new orbifold structure, $(L, l)$, on the line bundle $L$ using the following action of $G$ : for any $g \in G$ and $v \in L$

$$
l(g)(v)=\lambda(g) \cdot \bar{\rho}(g)(v) .
$$

Clearly for two different characters $\lambda$ and $\lambda^{\prime}$ the corresponding orbifold structures $l$ and $l^{\prime}$ on $L$ are different. It can be checked that any orbifold structure on $L$ is gotten this way.

The set of all isomorphism classes of orbifold bundles of the form $(L, \bar{\rho})$, where $L \in \operatorname{Pic}^{0}(Y)$, is denoted by $P_{G}^{\prime}(Y)$. Let $\hat{G}$ be the group of characters of $G$. We put down the summary of the previous discussions in the form of the following:

Lemma 2.2. The finite group of characters $\hat{G}$ acts freely on the group $P_{G}^{\prime}(Y)$, with the quotient being $\operatorname{Pic}_{G}(Y)^{\prime}$. The Lie group $\operatorname{Pic}_{G}(Y)^{\prime}$ is a finite index subgroup of the abelian group $\operatorname{Pic}_{G}(Y)$.

Now we want to determine the tangent space of $P_{G}^{\prime}(Y)$. From the above lemma it follows that for $(L, \bar{\rho}) \in P_{G}^{\prime}(Y)$, the tangent space $T_{(L, \bar{\rho})} P_{G}^{\prime}(Y)$ is canonically isomorphic to $T_{L} \operatorname{Pic}_{G}(Y)^{\prime}=T_{L} \operatorname{Pic}_{G}(Y)$.

There is an obvious lift of the action of $G$ to the trivial bundle $Y \times \mathbb{C}$. Let $H^{1}(Y, \mathcal{O})^{G}$ be the space of invariants of $H^{1}(Y, \mathcal{O})$, i.e., the subspace of $H^{1}(Y, \mathcal{O})$ on which $G$ acts trivially.

Lemma 2.3. The Lie algebra of the complex abelian Lie group $P_{G}^{\prime}(Y)$ is canonically isomorphic to $H^{1}(Y, \mathcal{O})^{G}$.

Proof. The action of the group $G$ on $Y$ induces a homomorphism

$$
\hat{\rho}: G \longrightarrow \operatorname{Aut}\left(\mathrm{Pic}^{0}(Y)\right) \text {; }
$$

$\operatorname{Aut}\left(\operatorname{Pic}^{0}(Y)\right)$ is the group of all automorphisms of the group $\operatorname{Pic}^{0}(Y)$. Now $\operatorname{Pic}_{G}(Y) \subset \operatorname{Pic}^{0}(Y)$ is the subgroup which is pointwise invariant under $\hat{\rho}(G)$. The Lie algebra of $\operatorname{Pic}^{0}(Y)$ is $H^{1}(Y, \mathcal{O})$. So the Lie algebra of $\operatorname{Pic}_{G}(Y)$ is $H^{1}(Y, \mathcal{O})^{G}$. But the Lie algebras of $\operatorname{Pic}_{G}(Y)$ and $P_{G}^{\prime}(Y)$ are isomorphic (follows from Lemma 2.2). This completes the proof. 
The following restriction is imposed on the group action:

Assumption. The quotient $X:=Y / G$ is a smooth variety. The quotient map $Y \longrightarrow X$, which is a morphism between smooth varieties, is denoted by $\pi$.

Let $(L, \bar{\rho})$ be an orbifold bundle on $Y$. Consider the direct image sheaf $\pi_{*} L$. Since $\pi$ is finite and flat, $\pi_{*} L$ is a locally free $\mathcal{O}_{X}$-coherent sheaf. The action $\bar{\rho}$ on $L$ induces a homomorphism of $G$ into $\operatorname{Aut}\left(\pi_{*} L\right)$, the automorphism group of the bundle $\pi_{*} L$. This homomorphism is denoted by $\rho^{\prime}$. Let $L^{G} \subset \pi_{*} L$ be the space of invariants, i.e., the subsheaf on which $G$ acts trivially. Clearly $L^{G}$ is a $\mathcal{O}_{X}$ submodule of $\pi_{*} L$. The homomorphism

$$
v \longmapsto \frac{1}{\# G} \sum_{g \in G} \rho^{\prime}(g)(v) \in \pi_{*} L
$$

defines a projection $\phi: \pi_{*} L \longrightarrow L^{G}$. In particular, the following exact sequence of $\mathcal{O}_{X}$-coherent sheaves on $X$

$$
0 \longrightarrow L^{G} \longrightarrow \pi_{*} L \longrightarrow \pi_{*} L / L^{G} \longrightarrow 0
$$

splits, and $L^{G}$ is a line subbundle of $\pi_{*} L$.

The higher direct images of $\pi$ vanish and $H^{i}(Y, L)$ is canonically isomorphic to $H^{i}\left(X, \pi_{*} L\right)$. Also any $i$ th cocycle of $\pi_{*} L$ is a sum of cocycles of $L^{G}$ and $\operatorname{ker}(\phi)$. Let $H^{i}(Y, L)^{G} \subset H^{i}(Y, L)$ be the space of invariants. We have proved the following:

Lemma 2.4. The inclusion of sheaves $L^{G} \longrightarrow \pi_{*} L$ induces an isomorphism between $H^{i}\left(X, L^{G}\right)$ and $H^{i}(Y, L)^{G}$.

2b. Parabolic line bundles. Let $X$ be a connected smooth projective variety over $\mathbb{C}$ of dimension $n$. Let $D$ be a divisor of normal crossing on $X$. By this we mean that $D$ is a reduced effective divisor and each irreducible component of $D$ is smooth and they intersect transversally. Let $D=\sum_{i=1}^{d} D_{i}$ be the decomposition into irreducible components. Following [MY] we define

Definition 2.5. A parabolic line bundle on on $(X, D)$ is a pair of the form

$$
\left(L,\left\{\alpha_{1}, \ldots, \alpha_{i}, \ldots, \alpha_{d}\right\}\right)
$$

where $L$ is a holomorphic line bundle on $X$ and any $0 \leq \alpha_{i}<1$ is a real numbers.

Assumptions. The weights $\left\{\alpha_{1}, \ldots, \alpha_{d}\right\}$ are fixed once and for all, and they are assumed to be nonzero rational numbers; in particular $\alpha_{i}=m_{i} / N$ for some integer $N$ (independent of $i$ ) and $1 \leq m_{i}<N$. For a divisor $D \subset X$ 
let $[D] \in H^{2}(X, \mathbb{Z})$ denote the Poincaré dual of $D$. It is assumed that the element $\sum_{i=1}^{d} \alpha_{i}\left[D_{i}\right] \in H^{2}(X, \mathbb{Q})$ belongs to the image of $H^{2}(X, \mathbb{Z})$.

Notation. Let $P(X)$ denote a component of the moduli space of holomorphic isomorphism classes of line bundles on $X$ with first Chern class $\sum_{i=1}^{d}-\alpha_{i}\left[D_{i}\right]$. (From the assumption and Lefschetz 1-1 theorem it follows that $P(X)$ is non-empty.)

The "Covering Lemma" (Theorem 1.1.1 of [KMM], Theorem 17 of $[\mathrm{K}]$ ) says that there is a connected smooth projective variety $Y$ and a finite Galois morphism

$$
\pi: Y \longrightarrow X
$$

with Galois group $G=\operatorname{Gal}(\operatorname{Rat}(Y) / \operatorname{Rat}(X))$ such that $\tilde{D}:=\left(\pi^{*} D\right)_{\text {red }}$ is a divisor of normal crossing on $Y$ and $\pi^{*} D_{i}=k_{i} N\left(\pi^{*} D_{i}\right)_{r e d}, 1 \leq i \leq d$, where $k_{i}$ are positive integers.

Define $\tilde{D}_{i}:=\left(\pi^{*} D_{i}\right)_{\text {red }}$; so $\pi^{*} D_{i}=k_{i} N \tilde{D}_{i}$. The divisor $\pi^{*} D_{i}$ is obviously invariant under the action of the Galois group $G$ on $Y$, and hence, the reduced divisor $\tilde{D}_{i}$ is also invariant under the action. In particular, the line bundle $\mathcal{O}\left(\tilde{D}_{i}\right)$ has an orbifold structure. For any $k \in \mathbb{Z}$ the bundle $\mathcal{O}\left(k \tilde{D}_{i}\right)$ has an induced orbifold structure.

Let $\xi \in P(X)$. The pull-back bundle $\pi^{*} \xi$ has an obvious orbifold structure. Define

$$
L:=\pi^{*}(\xi) \otimes \mathcal{O}\left(\sum_{i=1}^{d} k_{i} m_{i} \tilde{D}_{i}\right) .
$$

This line bundle $L$ has an orbifold structure

$$
c_{1}(L)=\pi^{*} c_{1}(\xi)+\sum_{i=1}^{d} k_{i} m_{i}\left[\tilde{D}_{i}\right]=\pi^{*} c_{1}(\xi)+\sum_{i=1}^{d} \frac{m_{i}}{N}\left[k_{i} N \tilde{D}_{i}\right] .
$$

By definition, $\left[k_{i} N \tilde{D}_{i}\right]=\pi^{*}\left[D_{i}\right]$, so

$$
c_{1}(L)=\pi^{*} c_{1}(\xi)+\sum_{i=1}^{d} \frac{m_{i}}{N} \pi^{*}\left[D_{i}\right]=0 .
$$

Hence, $L \in \operatorname{Pic}^{0}(Y)$. For a general point $p$ of $\tilde{D}_{i}$, the isotropy group is the cyclic group $\mathbb{Z} /\left(k_{i} N\right)$. The action of any $n \in \mathbb{Z} /\left(k_{i} N\right)$ on the fiber $L_{y}$ is multiplication by $\exp \left(2 \pi \sqrt{-1} n m_{i} / N\right)$.

Let $\hat{D} \subset Y$ be the reduced effective divisor consisting of all the points $y \in Y$ such that the isotropy group of $y$ for the $G$ action is nontrivial. That $\hat{D}$ is a divisor follows from the assumption that $X$ is smooth. (The 
bundle map $d \pi: \pi^{*} \Omega_{X}^{1} \longrightarrow \Omega_{Y}^{1}$ fails to be an isomorphism precisely over $\hat{D}$.) So $\tilde{D}$ is contained in $\hat{D}$.

Recall the group $P_{G}^{\prime}(Y)$ defined in Section 2a. Let $P_{G}(Y) \subset P_{G}^{\prime}(Y)$ be the set of all orbifold bundles $\bar{L}$ such that for a general point $p$ of $\tilde{D}_{i}$, the action of $n \in \mathbb{Z} /\left(k_{i} N\right)$ (the group $\mathbb{Z} /\left(k_{i} N\right)$ is the isotropy group of $p$ ) on the fiber $\bar{L}_{p}$ is multiplication by $\exp \left(2 \pi \sqrt{-1} n m_{i} / N\right)$, and on a general point, $y$, of any other component of $\hat{D}$ (not in $\tilde{D}$ ) the action of the isotropy group of $y$ on $\bar{L}_{y}$ is trivial. From rigidity of the representations of a finite group it follows that $P_{G}(Y)$ is both open and closed in $P_{G}^{\prime}(Y)$.

Define the morphism $F: P(X) \longrightarrow P_{G}(Y)$ using the correspondence $\xi \mapsto L$ obtained above.

Theorem 2.7. The morphism $F: P(X) \longrightarrow P_{G}(Y)$ is an isomorphism.

Proof. For $L \in P_{G}(Y)$ let $L^{G}$ be the line bundle on $X$ gotten by taking the invariant direct image (as done in Section $2 \mathrm{a}$ ).

Let $U:=\{z \in \mathbb{C}|| z \mid<1\}$ be the open disk and $U \times \mathbb{C}$ be the trivial line bundle on $U$. Let the group $\mathbb{Z} /(m n)$ act on $U$ by $\alpha \circ z=$ $\exp (2 \pi \sqrt{-1} \alpha /(m n)) z$, where $\alpha \in \mathbb{Z} /(m n)$ and $z \in U$, and let $\mathbb{Z} /(m n)$ act on $U \times \mathbb{C}$ by

$$
\alpha \circ(z, c)=(\exp (2 \pi \sqrt{-1} \alpha /(m n)) z, \exp (2 \pi \sqrt{-1} \alpha / m) c) .
$$

Then the pull-back of the line bundle on $U /(\mathbb{Z} /(m n))$ to $U$, given by the $\mathbb{Z} /(m n)$-invariant sections, is generated as an $\mathcal{O}(U)$-module by the section $\left(z, z^{n}\right)$ of $U \times \mathbb{C}$. This observation implies that $L$ is isomorphic to $\pi^{*}\left(L^{G}\right) \otimes \mathcal{O}\left(\sum_{i=1}^{d} k_{i} m_{i} \tilde{D}_{i}\right)$. So

$$
c_{1}\left(\pi^{*} L^{G}\right)=c_{1}(L)-\sum_{i=1}^{d} k_{i} m_{i}\left[\tilde{D}_{i}\right]=-\sum_{i=1}^{d} \frac{m_{i}}{N} \pi^{*}\left[D_{i}\right],
$$

so $L^{G} \in P(X)$. Thus the correspondence $L \mapsto L^{G}$ gives a morphism

$$
F^{\prime}: P_{G}(Y) \longrightarrow P(X) .
$$

Assume that $\xi \in P(X)$ and $L$ are related as in (2.6). The divisor $\tilde{D}_{i}$, $1 \leq i \leq d$, is effective, so there is an inclusion of sheaves $j: \pi^{*}(\xi) \longrightarrow$ $\pi^{*}(\xi) \otimes \mathcal{O}\left(\sum_{i=1}^{d} k_{i} m_{i} \tilde{D}_{i}\right)$. Moreover this homomorphism $j$ commutes with the actions of $G$ on $\pi^{*} \xi$ and $\left(\pi^{*} \xi\right) \otimes \mathcal{O}\left(\sum_{i=1}^{d} k_{i} m_{i} \tilde{D}_{i}\right)$. So $j$ induces a homomorphism $j^{\prime}: \pi^{*} \xi \longrightarrow \pi^{*} L^{G}$. This homomorphism, being $G$-equivariant, induces a homomorphism $\bar{j}: \xi \longrightarrow L^{G}$. However,

$$
c_{1}(\xi)=-\sum_{i=1}^{d} \frac{m_{i}}{N}\left[D_{i}\right]=c_{1}\left(L_{G}\right)
$$


so $\bar{j}$ must be an isomorphism. Thus we have proved that $F^{\prime} \circ F=I d$. We saw earlier that $L=\pi^{*}\left(L^{G}\right) \otimes \mathcal{O}\left(\sum_{i=1}^{d} k_{i} m_{i} \tilde{D}_{i}\right)$. This implies that $F \circ F^{\prime}=I d$, completing the proof.

Remark 2.8. $P(X)$ is irreducible. So Theorem 2.7 implies that $P_{G}(Y)$ is also irreducible. Neither $P_{G}(Y)$ nor $P(X)$ have Lie group structure. But they have affine group structure. The variety $P(X)$ is an affine group for the group $\operatorname{Pic}^{0}(X)$. Let $\bar{P}_{G}(Y) \subset P_{G}^{\prime}(Y)$ be the subgroup of all orbifold bundles $\bar{L}$ such that for a general point $p$ of $\tilde{D}_{i}$, the action of the isotropy group of $p$ on the fiber $\bar{L}_{y}$ is trivial. Clearly $P_{G}(Y)$ is an affine group for the group $\bar{P}_{G}(Y)$, where the action is given by tensor product. From Theorem 2.7 it follows that the two abelian groups $\operatorname{Pic}^{0}(X)$ and $\bar{P}_{G}(Y)$ are canonically isomorphic. Using this isomorphism, the morphism $F$ in Theorem 2.7 is an isomorphism of affine groups.

\section{Proof of Theorem A}

We continue with the notation of the previous section.

Define the subvariety $S_{m}^{i}:=\left\{(L, \bar{\rho}) \in P_{G}(Y) \mid \operatorname{dim} H^{i}(Y, L)^{G} \geq m\right\}$. We want to prove the following:

Theorem 3.1. Any irreducible component of $S_{m}^{i}$ is a translation of an abelian subvariety of $\bar{P}_{G}(Y)$ by a point of $P_{G}(Y)$ for the action defined in Remark 2.8.

The special case of the above theorem where $G=\{e\}$ was proved in [GL2]. It is easy to see that the proof in [GL2] goes through verbatim in our situation. (The Theorem 1.6 of [GL1] is one of the key points in the proof in [GL2]. The equivariant analogue of [Theorem 1.6 GL1] is also easily seen to be true.) We refrain from reproducing the argument in [GL2].

Define

$$
T_{m}^{i}:=\left\{L \in P(X) \mid \operatorname{dim} H^{i}(X, L) \geq m\right\} .
$$

Lemma 2.4 implies that the isomorphism $F$ between $P(X)$ and $P_{G}(Y)$, obtained in Theorem 2.7, identifies the subvariety $S_{m}^{i}$ of $P_{G}(Y)$ with the subvariety $T_{m}^{i}$ of $P(X)$.

After choosing a base point $q \in P(X)$ the variety $P(X)$ is identified with the abelian variety $\operatorname{Pic}^{0}(Y)$. Given a subvariety $V \subset P(X)$, whether it is a translation of an abelian subvariety does not depend upon the choice of the base point $q$. In Remark 2.8 we saw that the two affine group structures of $P_{G}(Y)$ and $P(X)$ coincide. So Theorem 3.1 gives the Thereon A stated in the introduction. 


\section{References}

[Bi] I. Biswas, A Remark on a deformation theory of Green and Lazarsfeld, J. reine angew. Math. 449 (1994), 103-124.

[GL1] M. Green and R. Lazarsfeld, Deformation theory, generic vanishing theorems, and some conjectures of Enriques, Catanese and Beauville, Invent. Math. 90 (1987), 389-407.

[GL2] M. Green and R. Lazarsfeld, Higher obstructions to deforming cohomology groups of line bundles, J. Amer. Math. Soc. 4 (1991), 87-103.

[K] Y. Kawamata, Characterization of the abelian varieties, Compositio Math. 43 (1981), 253-276.

[KMM] Y. Kawamata, K. Matsuda and K. Matsuki, Introduction to the minimal model problem, Adv. Stu. Pure Math. 10 (1987), 283-360.

[MY] M. Maruyama and K. Yokogawa, Moduli of parabolic stable sheaves, Math. Ann. 293 (1992), 77-99.

[M] D. Mumford, On the equations defining abelian varieties, I. Invent. Math. 1 (1966), 287-354.

School of Mathematics, Tata Institute of Fundamental Research, Homi BhabHa Road, Bombay 400005, INDIA

Current address: Institut Fourier, 100 rue des Maths, B.P. 74, 38402 Saint-Martind'Hères Cedex, FRANCE

E-mail address: indranil@math.tifr.res.in, biswas@puccini.ujf-grenoble.fr 